\title{
Online Balanced Motion Generation for Humanoid Robots
}

\author{
Grzegorz Ficht and Sven Behnke
}

\begin{abstract}
Reducing the complexity of higher order problems can enable solving them in analytical ways. In this paper, we propose an analytic whole body motion generator for humanoid robots. Our approach targets inexpensive platforms that possess position controlled joints and have limited feedback capabilities. By analysing the mass distribution in a humanoid-like body, we find relations between limb movement and their respective CoM positions. A full pose of a humanoid robot is then described with five point-masses, with one attached to the trunk and the remaining four assigned to each limb. The weighted sum of these masses in combination with a contact point form an inverted pendulum. We then generate statically stable poses by specifying a desired upright pendulum orientation, and any desired trunk orientation. Limb and trunk placement strategies are utilised to meet the reference CoM position. A set of these poses is interpolated to achieve stable whole body motions. The approach is evaluated by performing several motions with an igus ${ }^{\circledR}$ Humanoid Open Platform robot. We demonstrate the extendability of the approach by applying basic feedback mechanisms for disturbance rejection and tracking error minimisation.
\end{abstract}

\section{INTRODUCTION}

For humanoid robots to be able to operate in a human environment, a wide array of motoric skills is necessary, where keeping the robot balanced is of utmost importance. This is particularly difficult due to the possible mechanical inaccuracies, inexact actuation, and inaccurate sensor feedback. The impact of these factors varies between robots, and is highly correlated with their price. Smaller, low-cost platforms, such as the widely used Aldebaran Nao, can cope better with these limitations-due to a comparatively high torque to weight ratio, which allows them to ignore several kinematic and dynamic constraints when performing an action. This is often not the case with larger-sized platforms possessing a lower torque to weight ratio, which require more sophisticated control approaches. The current state-of-the-art however, focuses mostly on expensive high-quality robots, which rely on high control loop frequencies, precise torquecontrolled actuators, and a considerable amount of feedback information. Often, numerical optimisation methods are used for their control. Simplifying the complex kinematic models can enable analytic solutions.

In this paper, we present an analytic method for run-time generation of parametric quasi-static motions from inherently balanced static pose keyframes, based on the concept of triangle centroid mass (see Fig. 1), The limbs and whole body of a robot are described with triangles, which allows

All authors are with the Autonomous Intelligent Systems (AIS) Group, Computer Science Institute VI, University of Bonn, Germany. Email: ficht@ais. uni-bonn. de. This work was partially funded by grant BE 2556/13 of the German Research Foundation (DFG).

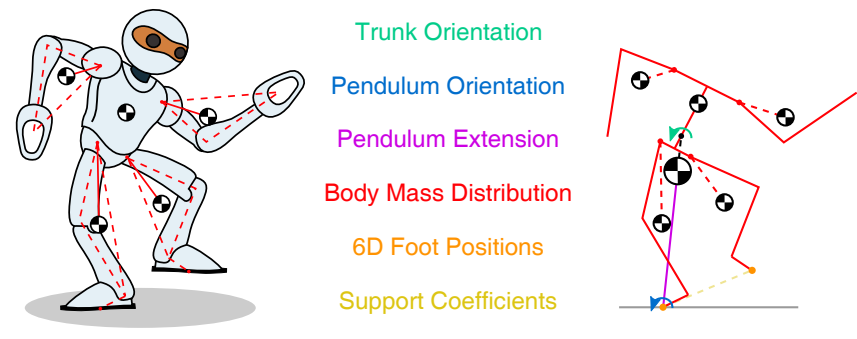

Fig. 1. Approximation of a humanoid body and its limbs with triangles. The approximation allows to parameterise a whole body pose with only a few parameters to produce balanced motions.

for finding a direct mapping between body movement and its respective CoM location. The proposed method is applicable to larger robots, equipped with inexpensive actuators and sensors. By reducing the complexity of the model, we are able to make the robot balance in various configurations without any information about the forces or torques. We rely exclusively on an on-board 6-axis IMU and joint positions.

\section{RELATED WORK}

The first motion generation methods for humanoid robots pre-computed joint trajectories with little online modifications. Hirai et al. [1] for example, derived a reference body trajectory based on the desired Zero Moment Point (ZMP) and generated joint angle displacements to shift the ZMP to an appropriate position to maintain balance while walking with the Honda P2 platform. A different approach to this problem has been proposed by Fujimoto et al. [2], who perform the planning and control in a combined manner to track the reference Center of Mass (CoM) trajectory with respect to ground reaction forces in a real-time simulated environment. These works focus on solving the single task of generating motions to keep a humanoid robot balanced while walking.

Methods of generating whole-body motions for humanoids in a more general way have been developed by Kajita et al. in [3]. Given reference linear and angular momenta while considering the constraints presented by contacts, a balanced kicking and walking motion was generated and performed by the HRP-2 robot through joint velocity control. A different approach to the same problem was developed by Sentis and Khatib [4], where the control of lower priority tasks is projected into the null-space of the higher priority ones. The proposed hierarchical framework addresses a large set of constraints and provides compliant torque-based control that allows humanoid robots to simultaneously perform multiple tasks of varying complexity. These two motion generation techniques have led to the emergence of multiple Whole- 
Body Control (WBC) systems utilising joint velocities or torques to generate and perform motions. Position-controlled actuators are not addressed by these control schemes. Although it is possible to integrate the results to achieve a desired position, due to the accumulated noise, these approaches are hardly applicable on low-cost, real robots.

The output produced by the Whole-Body Controller is only one way to categorise them. Another way of distinction is by the method of finding a solution, which can be divided into closed-form and optimisation-based techniques. The former use a series of mathematical operations to achieve a desired joint trajectory, as in the mentioned approaches [3], [4]. In the latter, the result is computed by a solver from a user-defined optimisation problem, which is generally more time consuming. Many recent works [5], [6], [7] are focused on using solvers, as they allow for finding solutions to possibly conflicting tasks. The long computation time, however often makes these types of methods unfavorable when it comes to using them online on real robots, as they cannot be run at interactive rates. To remedy this, Dai et al. [8] use the robot's full kinematics with reduced dynamics, as the full dynamics did not allow the solver to converge even after days of computations. With the proposed approach they are able to interactively generate motions in less than $0.2 \mathrm{~s}$.

Reducing the complexity of a humanoid robot representation, while retaining most of the relevant information for control purposes led to the development of various models. Kajita et al. [9] introduced the 3D Linear Inverted Pendulum, which allowed for generation of a biped walk depending on desired velocity and direction. In this model, the mass stays at a constant height and a change in the robot's configuration cannot influence it by definition. The Reaction Mass Pendulum described by Lee et al. [10] is a more comprehensive model, that uses three pairs of equal point masses at different radial distances to shape the inertia of the robot. These masses are abstract however, and do not map directly to a specific limb or body part. Describing the robot by distributed masses was also done by Takenaka et al. [11] for the purpose of fast gait generation. Their simplified model allows for fast calculations, but does not consider upper body movement and kinematic constraints.

Regardless of how the joint trajectories were generated, specification of a stability criterion is necessary to keep the robot balanced during a motion. Most of the current developments make certain that the ZMP lies inside the support polygon, be it the original definition [12], or a more generalised one [13]. A universal method of assessing stability was also presented by Hirukawa et al. in [14], where stability is judged by whether the gravity and inertia wrench lies inside the polyhedral convex cone of the contact wrench between the robot and its environment.

To properly evaluate the stability through e.g. the ZMP, Whole-Body Control techniques rely on good sensor feedback. This limits their usability to higher-quality hardware where force-torque sensors are available. The ZMP could also be estimated through the use of inverse dynamics, but that again requires accurate feedback data as even small noise can greatly influence the result. These factors are frequently an issue with lower cost platforms, and in combination with an unfavorable torque to weight ratio, the applicability of WBC on them is highly restricted.

\section{Model CompleXity Reduction}

When considering a humanoid body plan, the CoM is not fixed to any of the links, but rather moves in a certain region depending on the placement of body parts. This region lies in the vicinity of the lower part of the trunk-as it is the heaviest part of the body with 50 to 55 percent of the total mass, following with the legs (from 30 to 35 percent) [15]. In any standing pose scenario, the legs perform a supporting function by connecting the hipline to the contacting surface (ground). If the contact is approximated by a single origin point $O$, the spine is stiff and begins at the hipline midpoint $H$ and ends at the shoulder midpoint $S$, then it is possible to describe such a pose by a triangle connecting these points $\triangle O S H$. If the body is symmetrical, the resulting CoM will lie inside of this triangle, with its exact position depending on the mass distribution between all body parts. This position can be considered as nominal and(depending on limb movement) can still change. The contact point and CoM form a pendulum that can be used to describe the stability of the robot. We consider the neck and head as a part of the trunk, as the limited motion it can achieve paired with the relatively low weight does not influence the CoM in a significant way. Furthermore, unconstrained head movement is desirable for the vision system. These assumptions are the basis of the work described in this section.

Other than a humanoid's whole body pose being represented by a triangle, the same characteristic can be observed in the arms and legs (Fig. 1). By placing points at the hips $H^{L}, H^{R}$, knees $K^{L}, K^{R}$ and ankles $A^{L}, A^{R}$ for the legs as well as the shoulders $S^{L}, S^{R}$, elbows $E^{L}, E^{R}$, and wrists $W^{L}, W^{R}$ for the arms, four triangles can be defined with an accompanying CoM that lies within them. These limb triangles have one side with variable length, depending on the extension of the limb and two sides with known and fixed lengths. In the arms, these are the upper arm and forearm while in the leg they are represented by the thigh and shank. By parameterising the mass distribution in the limbs, it is possible to associate joint angles and the limb mass placement in space with a direct mapping (see Section IIIA). As with the head and the trunk, we assume the feet and hands do not influence the CoM of their respective limbdue to their limited range of movement and comparatively low weight.

With these assumptions, it is possible to describe a pose of a humanoid robot through the use of five point masses, where the main one is attached to the torso, and the remaining four are assigned to the limbs. The trunks centre of mass is described with a three-dimensional offset from the shoulder midpoint. The origins of the limbs are located at the shoulders and hips, which are spaced symmetrically away from their respective midpoints at exactly half the shoulder width $s_{w}$ and hip width $h_{w}$, accordingly. The whole body mass 


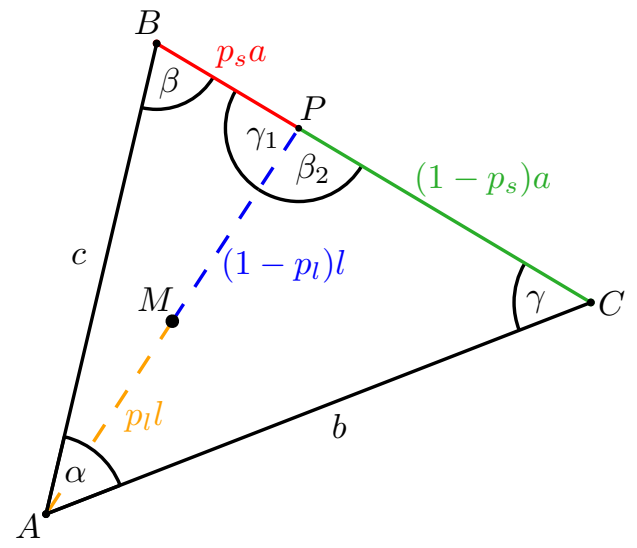

Fig. 2. Mass distribution in a triangle. Knowing the distribution parameters $p_{s}, p_{l}$ and two sides $a, c$, it is possible to calculate all of the angles, given a desired mass placement $M$ in respect to origin $A$.

is a weighted sum of the five body part mass coordinates, and forms a pendulum with the single ground contact point. The proposed approximation might not provide any benefit when doing a forward calculation of the CoM based on joint positions and physical properties of the links. The inverse operation of placing the robots CoM in space however, is greatly simplified and allows to utilise analytical limb mass placement strategies that do not involve optimisation. Generating a pose based on the desired CoM position is the main contribution of the presented work and is described in detail in Section IV. Due to the assumptions made, it is possible that some accuracy with regard to CoM tracking may be lost. In Section VI we demonstrate that this effect is minor.

\section{A. Centroidal mass in a triangle}

Given a triangle with uniform density, the centroid is located at the intersection of its three medians, however only two of them are necessary to determine it. The centroid divides each of the medians with a ratio of $2: 1$, meaning that it is located $2 / 3$ of the distance from any selected vertex to the midpoint of the side opposing it. The location of the mass center can therefore be described with as little as two values: $p_{s}$ and $p_{l}$-named the CoM distribution parameters. $p_{s}$ is the ratio of chosen side $s$ at which the line segment $l$ from the opposing vertex $v$ sections it to its length, and $p_{l}$ is the ratio of the distance from the same vertex $v$ along line $l$ at which the CoM is located to that line's length. In a regular triangle $p_{s}=\frac{1}{2}$, while $p_{l}=\frac{2}{3}$, and line $l$ is a median. By manipulating these parameters, it is possible to place the CoM anywhere inside the triangle in accordance to its mass distribution.

Let us consider an example in two-dimensional space to depict the possible solution. Given a desired CoM position $M$ in regards to the origin $A$ with two known sides $a, c$ and the CoM distribution parameters $p_{s}, p_{l}$, compute the missing angles $\alpha, \beta, \gamma$ and the length of side $b$ in triangle $\triangle A B C$ as shown on Fig. 2. The line segment $l$ dividing $\triangle A B C$ at point $P$ into two smaller triangles $\triangle A B P, \triangle A P C$ can be computed as

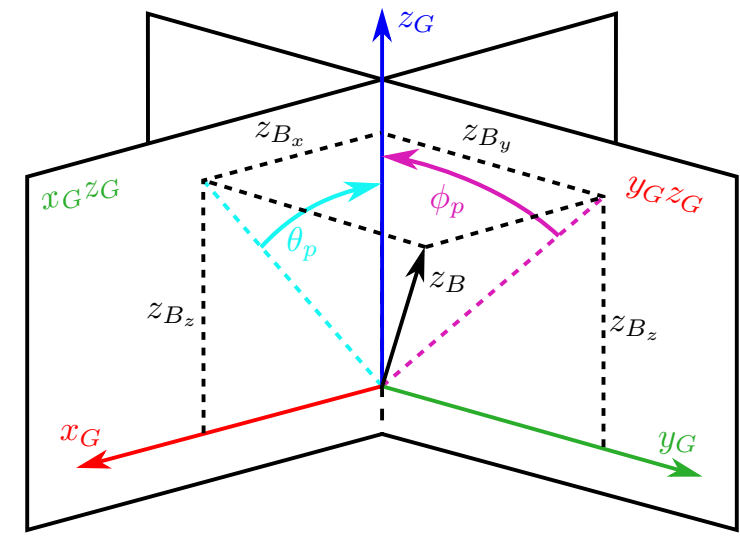

Fig. 3. Projected angles definition. A combination of two 2D angles $\theta_{p}, \phi_{p}$ on perpendicular planes $x_{G} z_{G}, y_{G} z_{G}$ is used to define a $3 \mathrm{D}$ orientation of a body's z-axis $z_{B}$.

$$
l=d(A, M) / p_{l},
$$

where $d(A, M)$ is the Euclidean distance between points $A$ and $M$. From the law of cosines, we compute the relations in triangles $\triangle A B P, \triangle A P C$ :

$$
\begin{aligned}
& c^{2}=l^{2}+\left(p_{s} a\right)^{2}-2 p_{s} l \cos \left(\gamma_{1}\right), \\
& b^{2}=l^{2}+\left(\left(1-p_{s}\right) a\right)^{2}-2\left(1-p_{s}\right) l \cos \left(\pi-\gamma_{1}\right) .
\end{aligned}
$$

For a cleaner notation let us introduce a complementary ratio $p_{c}=1-p_{s}$, and knowing that $\cos \left(\pi-\gamma_{1}\right)=-\cos \left(\gamma_{1}\right)$, we can solve for $b$ :

$$
b=\sqrt{2 l^{2}+p_{c}^{2} a^{2}+p_{s}^{2} a^{2}+2\left(p_{c}-p_{s}\right) l a \cos \left(\gamma_{1}\right)-c^{2}} .
$$

Angle $\gamma_{1}$ in triangle $\triangle A B P$ can be computed as

$$
\begin{aligned}
\alpha_{1} & =\operatorname{acos}\left(\frac{-p_{s}^{2} a^{2}+l^{2}+c^{2}}{2 l c}\right), \\
\beta_{1} & =\operatorname{acos}\left(\frac{p_{s}^{2} a^{2}-l^{2}+c^{2}}{2 p_{s} a c}\right), \\
\gamma_{1} & =\pi-\alpha_{1}-\beta_{1} .
\end{aligned}
$$

Finally, having all side lengths of triangle $\triangle A B C$, we compute its $\alpha, \beta, \gamma$ angles similarly as in Eqs. (5) to (7), by substituting $b$ for $l$ and $a$ for $p_{s} a$. The triangle describing the human pose in relation to the CoM of the whole body can be calculated in a similar manner as the one for the $\mathrm{CoM}$ of the limb with minor changes to the method. The difference stems from the fact that the triangle associated with the body has only a single side with invariable lengththe spine length. However, by knowing the orientation of the spine it is possible to compute its relative orientation $\gamma_{1}$ to the line segment $l$, which in turn allows to calculate the missing variables with the method above. This strongly relates to the trunk placement strategy, described in Section IV-A.

\section{B. Projected angles}

We extend the two-dimensional approach to three dimensions with the use of projected angles. Many parameterisations for rotations exist with their inherent properties depending on the definition as shown in [16], where the authors perform a review and summary of these and introduce 
two new representations - tilt angles and fused angles. In the same work, it is mentioned that new parameterisations are created to provide convenient exploits that increase the efficiency of algorithms or ease the geometric interpretation. We motivate the usage of projected angles with the need to set the inclination of objects when projected onto separate global $x z$ and $y z$ planes, in a way where an inclination on one plane does not influence the other. In this work, we assume a right-handed coordinate system, with the $\mathrm{x}$-axis pointing forward, $y$-axis pointing to the left of the robots origin and z-axis pointing upwards.

As with fused angles, we base our definition on the intermediate tilt angles representation, and introduce the projected pitch and projected roll angles. The definition of these projected angles is illustrated on Fig. 3. In fused angles, the $z_{G}$ vector is projected on the $y_{B} z_{B}$ and $x_{B} z_{B}$ planes which are fixed to the body, while projected angles perform the inverse operation of projecting the $z_{B}$ vector onto the global $y_{G} z_{G}$ and $x_{G} z_{G}$ planes, which define the projected pitch $\theta_{p}$ and projected roll $\phi_{p}$, respectively. The mathematical definitions are as follows:

$$
\begin{array}{ll}
\theta_{p}=\operatorname{atan} 2\left(z_{B x}, z_{B z}\right) & \in[-\pi, \pi], \\
\phi_{p}=\operatorname{atan} 2\left(z_{B y}, z_{B z}\right) & \in[-\pi, \pi] .
\end{array}
$$

The definition is completed with the fused yaw $\psi$, which performs the rotation around $z_{B}$, as in [16]. It can be observed that projected angles are nonlinear and have a singularity when at least one of the angles is equal to $-\frac{\pi}{2}$ or $\frac{\pi}{2}$. To accommodate for these problems, we use spherical interpolation in combination with a prohibition band of negligible width around the singularity values.

The main advantage of projected angles is the ability to specify the global orientation of objects where a projected inclination remains the same, regardless of the projection in the plane perpendicular to it. This is not the case when performing the rotations sequentially, or with fused angles. Another benefit of this representation is that specifying a desired global orientation of the spine in combination with a set pendulum orientation makes the computation of $\gamma_{1}$ in Eq. (7) straightforward in both the pitch and roll directions. The 3D solution is then equivalent to a pair of $2 \mathrm{D}$ solutions, greatly simplifying the calculations.

\section{BALANCED MOTION GENERATION}

With the model description introduced in Section III, we define a static whole body pose of a humanoid robot through a set of four components:

- Body pendulum: projected roll $\phi_{p}^{B}$, projected pitch $\theta_{p}^{B}$, heading $\omega^{B}$ and length $l^{B}$ encode the balance of a humanoid robot into a pendulum with mass $m^{B}$.

- Trunk orientation: projected roll $\phi_{p}^{T}$, projected pitch $\theta_{p}^{T}$ and fused yaw $\psi^{T}$ are responsible for orienting the trunk with a spine of length $l^{T}$ in 3D.

- Feet positions: 6D poses $F_{l}, F_{r}$ define the placement of the feet.

- Support coefficient: $c_{s}$ describes the weight distribution between the feet.
The first step requires to determine the origin of the body pendulum $O$. This is done through a combination of the desired foot positions and the support coefficient. The support coefficient represents the whole weight distribution between the legs. Shifting the whole weight from the right to the left foot is expressed by $c_{s} \in[0,1]-$ a value of $c_{s}=\frac{1}{2}$ means that both feet equally support the mass of the robot. This is equivalent to moving the body pendulum origin between the left and right foot contact points.

Once the origin point has been determined, we utilise the desired pendulum orientation and length to calculate the target CoM position:

$$
\operatorname{com}^{B}=z^{B} l^{B}+O
$$

where $z^{B}$ is a unit vector representing the $\mathrm{z}$-axis direction of the body pendulum, calculated as

$$
\begin{aligned}
z_{x}^{B} & =\tan \left(\operatorname{sgn}\left(\cos \left(\theta_{p}^{B}\right)\right) \theta_{p}^{B}\right) \\
z_{y}^{B} & =-\tan \left(\operatorname{sgn}\left(\cos \left(\phi_{p}^{B}\right)\right) \phi_{p}^{B}\right) \\
z_{z}^{B} & =\operatorname{sgn}\left(\cos \left(\theta_{p}^{B}\right)\right)
\end{aligned}
$$

This method can be used to calculate any point in $3 \mathrm{D}$ space with the use of projected angles. Through controlling the extension and orientation of the CoM similarly to a pendulum, we have the possibility to influence the stability of the robot. In our approach, we constrain the body pendulum to an upright orientation to generate inherently balanced static poses.

\section{A. Trunk placement}

The description of a humanoid robot pose through a triangle ties the orientation of the trunk with the state of the body pendulum. As mentioned in Section III-A, the line connecting the robot's origin with the total CoM extends further and intersects the spine. This intersection point is called the trunk pivot point $t_{p p}$ and defines the origin of rotation for the trunk. It's coordinates can be calculated as in (10), by substituting the length with $l^{B} / p_{l}^{B}$. By defining a desired trunk orientation in the pose, we compute the hip and shoulder origins through

$$
\left[\begin{array}{l}
H_{x y z}^{L} \\
H_{x y z}^{R} \\
S_{x y z}^{L} \\
S_{x y z}^{R}
\end{array}\right]=\left[\begin{array}{ccc}
0 & \frac{h_{w}}{2} & -p_{s}^{B} l^{T} \\
0 & -\frac{h_{w}}{2} & -p_{s}^{B} l^{T} \\
0 & \frac{s_{w}}{2} & \left(1-p_{s}^{B}\right) l^{T} \\
0 & -\frac{s_{w}}{2} & \left(1-p_{s}^{B}\right) l^{T}
\end{array}\right] R_{T}^{-1}+t_{p p}
$$

where $R_{T}$ is the rotation matrix of the trunk, calculated with [17] as per [16]. The achieved point coordinates are then rotated around the global z-axis, at the pendulums origin by the heading value $\omega^{G}$.

\section{B. Foot placement}

The foot support points are offset from their respective ankles, and are located at the geometric centre of the feet. Given a desired 6D foot pose and knowing the foot offsets, we can directly compute the desired ankle position. Although the distance between the hip and ankle positions is sufficient to compute the extension of the leg $\mathrm{CoM}$ from its origin, 
it does not provide full information for placing the CoM in 3D. For a full definition of the CoM placement, the leg rotation needs to be specified. To simplify the calculations, we assume that the ankle does not possess a yaw joint, and therefore the whole leg yaws at the hip. This is the case for most of the robotic platforms available currently on the market. This assumption is not necessary, but its lack would require an alternative method to specify the set leg yaw.

We compute the leg yaw as the angle $\psi^{L}$ around the leg's z-axis $n_{1}$ between a set of planes which normals represent the base $n_{2}$ and desired $n_{3}$ pointing directions of the leg's Xaxis. A unit vector created from the line segment connecting the hip to the ankle in combination with a fused yaw of zero is used to compute the $n_{1} \mathrm{Z}$-axis and $n_{2}$ base $\mathrm{X}$-axis direction. The desired $\mathrm{x}$-axis direction vector $n_{3}$ is given by

$$
n_{3}=\frac{n_{1} \times n_{y}}{\left\|n_{1} \times n_{y}\right\|},
$$

where $n_{y}$ is the normal of a plane passing through three points: the hip origin, desired ankle position, and an $x y$ offset from that position representing the desired yaw component (e.g. foot's $\mathrm{x}$-axis direction vector, knee point etc.). The final leg yaw is computed as

$$
\psi^{L}=\pi-\operatorname{atan} 2\left(\left(n_{2} \times n_{3}\right) \cdot n_{1}, n_{3} \cdot n_{2}\right),
$$

which allows to fully define the leg CoM position.

\section{Arm placement}

The arms play a vital role in balancing a humanoid robot. When walking or performing a wide array of motions (e.g. kicking a ball, jumping), the arms can correct for a certain amount of error between the desired and current CoM at any given time. This is also partially true when one of the arms has an assigned task (e.g. carrying or reaching an object) as the other arm can move freely. Its relatively low weight can however mitigate only slight CoM displacements. Assuming that the legs (LL, RL) and trunk (T) have been placed, a task has been assigned to the right arm (RA) and its position has been calculated in a similar manner to the legs, the left arm (LA) desired CoM position then is simply

$\operatorname{com}^{L A}=\frac{\operatorname{com}_{w}^{B}-\left(\operatorname{com}_{w}^{T}+\operatorname{com}_{w}^{L L}+\operatorname{com}_{w}^{R L}+\operatorname{com}_{w}^{R A}\right)}{m^{L A}}$

where $\operatorname{com}_{w}$ are the mass-weighted CoM coordinates. The distance between $S^{L}$ and $c o m^{L A}$ can be out of the movement range defined by the minimum $e_{\min }$ and maximum $e_{\max }$ CoM extension and needs to be altered to account for that. Following this, the desired robot CoM placement cannot be met and requires a corrective action from the trunk to satisfy it.

This claim still holds when both arms are free to move, accordingly with the possible range of movement and their relative mass. With two arms however, both of these parameters are greater, therefore the corrective actions have a stronger influence on the effective CoM position. In order to place both arms in a way that their single combined CoM satisfies the desired whole-body CoM position requirement, an arm placement strategy is needed. Naturally, this problem could be solved using optimisation with a set of constraints however, that would greatly increase the computation time. Knowing that in some cases the CoM displacement error cannot be brought to zero without modifying the trunk position, brings the problem back to that of whole body motion planning.

The proposed arm positioning strategy needs to provide a continuous solution comprised of two coordinates $\mathrm{com}^{R A}, \mathrm{com}^{L A}$ that satisfies the desired CoM placement $\operatorname{com}_{d}^{A}$ when possible, in respect to the arm's range of motion. Due to the symmetry of the whole body in the $x z$ plane, the obvious approach would involve placing $\mathrm{com}^{R A}, \mathrm{com}^{L A}$ equidistantly on a line that crosses $\operatorname{com}_{d}^{A}$ and is parallel to the trunks y-axis. The solution performs well only when the $y$ component of $\operatorname{com}_{d}^{A}$ relative to the trunk is close to zero, as the symmetrical motion of the arms counterbalances itself when hitting limits. In addition, this strategy requires that both arms move with the shifting of $\mathrm{com}^{A}$, which produces unnatural looking poses. We slightly modify this method by using a different direction vector of the line supposed to cross $\operatorname{com}_{d}^{A}$. For this, two shoulder spheres are created, that originate at $S^{L}, S^{R}$ and have a radius of $e_{\max }$. The third sphere is placed at $\operatorname{com}_{d}^{A}$, and represents the proximity of the solution to the shoulders, with a radius of

$$
r=\min \left(d\left(\operatorname{com}_{d}^{A}, S^{L}\right), d\left(\operatorname{com}_{d}^{A}, S^{R}\right)\right)+e_{\max } .
$$

The two intersection $i^{L}, i^{R}$ points between the proximity sphere and the shoulder spheres form the initial direction line, which is then shifted to cross $\operatorname{com}_{d}^{A}$. In case the proximity sphere does not intersect the further shoulder sphere, the second intersection point is substituted by the point on that sphere's surface nearest to $\operatorname{com}_{d}^{A}$. The final direction line is then adjusted to account for $e_{\max }$ and a minimum $y$ distance of the arms from the trunk to avoid selfcollisions. The strategy used produces natural looking poses. As the movement of both arms is bound to the direction line which moves with $\operatorname{com}_{d}^{A}$ and with respect to arm extension limits, the produced result is continuous.

\section{Performing motions}

A set of keyframes representing statically stable poses can be used to generate stable motions, when the influence of dynamics is negligible, which is the case when the motion is performed slowly. For this purpose, based on the motion duration and control frequency we linearly interpolate all of the pose parameters apart from the projected pitch $\theta_{p}$ and roll $\phi_{p}$. Due to their intrinsic nonlinearity, we compute pairs of intermediate projected angles from the interpolated $\mathrm{z}$-axis coordinates. These coordinates lay on an arc of a great circle connecting the start $z^{S}$ and end $z^{E}$ pose $\mathrm{z}$-axis orientation unit vector. The angle between $z^{S}$ and $z^{E}$ coordinates laying on the great circle plane

$$
\angle_{g c}=\operatorname{atan} 2\left(\left\|z^{S} \times z^{E}\right\|, z^{S} \cdot z^{E}\right)
$$

is linearly interpolated and used to rotate $z^{S}$ coordinates around the great circle plane normal $n_{g c}$. Care is also 

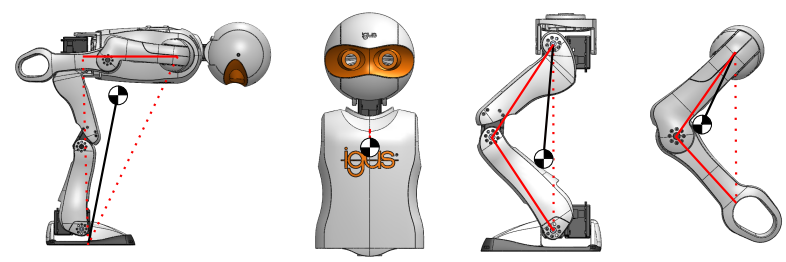

Fig. 4. Mass distribution in a CAD model of the igus ${ }^{\circledR}$ Humanoid Open Platform. From left to right: whole body, trunk, leg, and arm.

taken as to not wrap around $\pm \pi$ to avoid self-collisions and unwanted behaviour. In the majority of cases the result is a path on a minor arc which ensures minimum travel between the two pose orientations.

\section{CAlibration}

In order to use the method on a robot, a full body calibration to extract the $p_{s}$ and $p_{l}$ mass distribution parameters, $o_{x y z}^{T}$ trunk CoM offset and several body length measurements is necessary. For this purpose, a complete CAD model of the igus ${ }^{\circledR}$ Humanoid Open Platform robot was used [18]. The measurements, masses and CoM positions for each of the five body parts, as well as the complete robot's CoM position have been extracted from the CAD data and used without alteration.

In the igus ${ }^{\circledR}$ Humanoid Open Platform, the shoulders are $0.245 \mathrm{~m}$ apart, while the distance between the hips is $0.11 \mathrm{~m}$. The spine length, connecting the shoulder and hipline midpoints equals $0.225 \mathrm{~m}$. The limb's upper and lower links are symmetrical and possess lengths of $0.1995 \mathrm{~m}$ for the legs and $0.17 \mathrm{~m}$ for the arms. The geometric centers of the left and right foot are offset from their respective ankles by

$$
\begin{aligned}
& o_{x y z}^{L F}=\left(\begin{array}{lll}
0.035 & -0.011 & -0.038
\end{array}\right), \\
& o_{x y z}^{R F}=\left(\begin{array}{lll}
0.035 & 0.011 & -0.038
\end{array}\right)
\end{aligned}
$$

when the desired orientation of the foot remains vertical, and are subject to change with alterations to the foot orientation.

To retrieve the mass distribution information, we bent all of the limbs and the whole body at a right angle at their main joints. A $90^{\circ}$ angle formed at the hips, elbows and knees represents exactly half of the possible CoM extension, meaning that the calibration should provide equally good values for both $p_{s}$ and $p_{l}$. Calculating these parameters is straightforward, given the full limb triangle description and relative CoM position. An approximation of the resulting mass distribution is shown on Fig. 4.

Although averaging multiple measurements with different arrangements can be done to achieve more accurate results, we have found that a single calibration with a right angle formed by the fixed sides of the limbs yields a good approximation for $p_{s}$ and $p_{l}$. The trunk mass of $2.373 \mathrm{~kg}$ is offset from the shoulder midpoint by

$$
o_{x y z}^{T}=\left(\begin{array}{lll}
0.004 & 0 & -0.0475
\end{array}\right) .
$$

Due to the symmetry of the robot, the left and right limbs have the same mass and weight distribution. One arm weighs $0.554 \mathrm{~kg}$, while a leg has a mass of $1.332 \mathrm{~kg}$. The mass
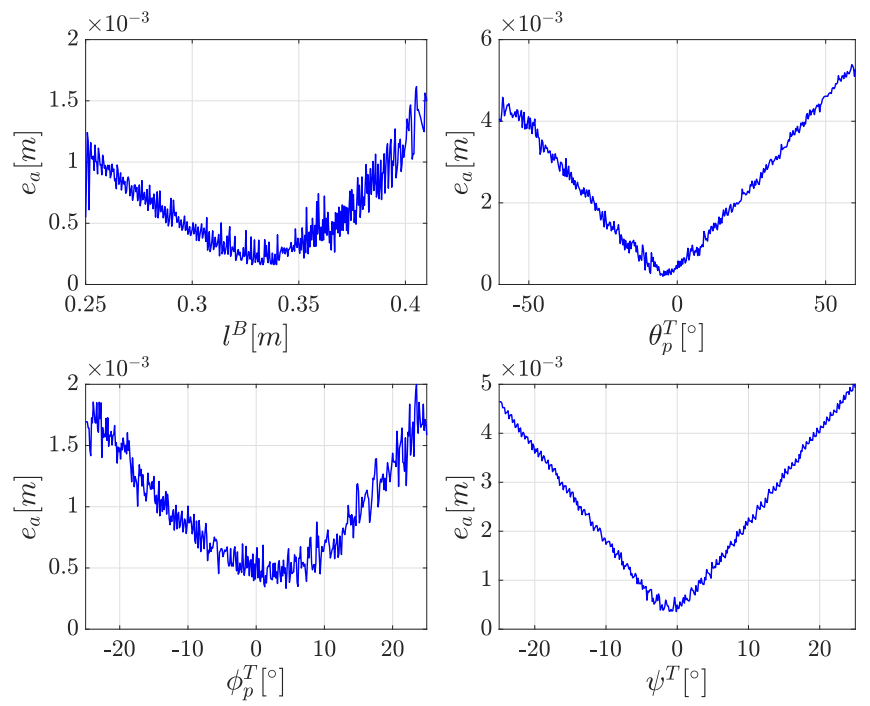

Fig. 5. Accuracy of the presented triangle approximation, with varying pendulum extension and trunk orientation parameters.

distribution parameters for the body, legs and arms used in our experiments are as follows:

$$
\left(\begin{array}{c}
p_{l}^{B} p_{s}^{B} \\
p_{l}^{L} p_{s}^{L} \\
p_{l}^{A} p_{s}^{A}
\end{array}\right)=\left(\begin{array}{ll}
0.7743 & 0.5479 \\
0.6159 & 0.7930 \\
0.5608 & 0.1664
\end{array}\right) .
$$

\section{EXPERIMENTAL RESUlTS}

Verification of the proposed method was carried out with an igus ${ }^{\circledR}$ Humanoid Open Platform robot. First, we evaluate the accuracy of the proposed approximation with the error between the CoM positions computed with our approach and the Universal Robotic Description Format (URDF) model for various poses of the robot. Next, we test the capability of performing whole body posing with single and double leg support poses, which we follow with performing a kicking motion. The achieved results are then quantified by the CoM tracking error.

\section{A. Approximation error}

To confirm that the assumptions made are correct, we measure the error $e_{a}$ between the CoM calculated with the URDF model (treated as ground truth) and the CoM calculated using our triangle approximation. A set of motions has been generated using the proposed method to assess the influence of the most critical parameters on the accuracythe pendulum extensions and trunk orientation. The first motion alters the body pendulum length $l^{B}$ across the whole possible range - from $0.25 \mathrm{~m}$ to $0.41 \mathrm{~m}$ with the trunk kept upright. The next motions keep $l^{B}$ constant at $0.30 \mathrm{~m}$, and vary the trunk orientation about each axis separately. The result of the approximation can be seen in Fig. 5 .

In a typical scenario where the trunk is upright, the error is generally below $1 \mathrm{~mm}$ and can reach as low as $0.2 \mathrm{~mm}$. The biggest contribution to the decrease in accuracy has the trunk orientation. As the trunk is the root part that connects all four of the limbs, their movement is paired with any change in the trunks orientation. As that movement breaks 

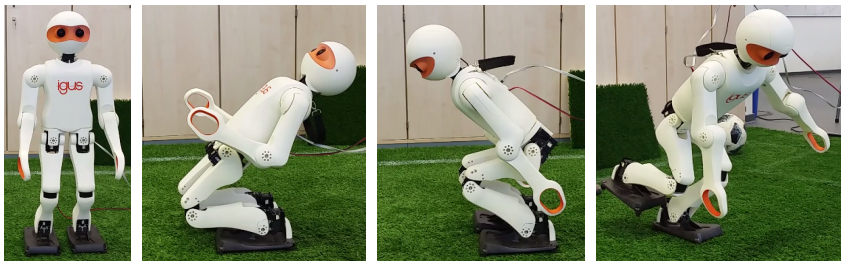

Fig. 6. The igus ${ }^{\circledR}$ Humanoid Open Platform in several balanced motion keyframes, with varying pendulum extension, trunk orientation, and support coefficient values.

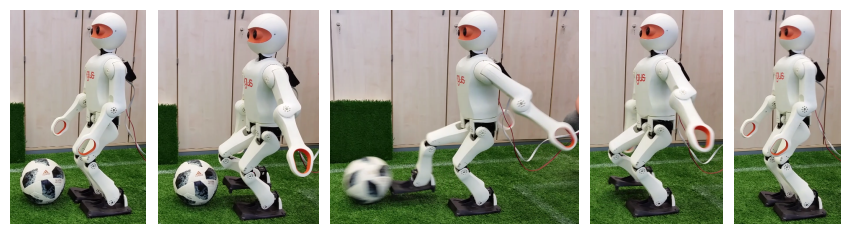

Fig. 7. Still frames from the generated kicking motions.

the symmetry, any error becomes quickly visible. The mass distribution parameters then become essential to proper CoM calculation and limb placement. This can be observed best with a change in the trunk projected pitch $\theta_{p}^{T}$ and fused yaw $\psi^{T}$ angles. The trunk projected roll $\phi_{p}^{T}$, also shows this property, but to a lesser extent. When the trunk is upright, all of the masses generally lie on the frontal plane and the error is mostly two-dimensional. Pitching or yawing the trunk moves the masses further away from the plane, increasing the errors. The error is symmetrical and grows linearly, which leads to suggest that it is an effect of a slight error in the calibration of the mass distribution parameters.

As the calibration was done with a single data point, the results can still be improved. One possibility of addressing this would involve averaging several data points at different joint configurations. Searching for an optimal set of parameters with an error minimising function is also feasible. In spite of the minor miscalibration, the errors in the possible range of movement do not exceed $5 \mathrm{~mm}$, which translates to a maximum error of $1.6 \%$. The achieved results are considered to be sufficiently accurate in order to apply the method on a real robot.

\section{B. Balanced whole body posing}

We verify our motion generator, with multiple test poses in single and double leg support with various trunk orientations; a sample of these can be observed in Fig. 6. The motions to reach these poses were generated at runtime by modifying the parameters introduced at the beginning of Section IV.

One noteworthy property to mention is that with the trunk and pendulum fully upright and a nominal pendulum extension, our method generates a natural looking standby pose, as seen on the left of Fig. 6. On the same figure it can be observed that even quite complex motions in terms of balance can be performed. This is a result of the inherent vertical orientation of the body pendulum when generating the pose.

\section{Kicking motion}

Generating a motion to achieve a balanced pose which is parametric is a very useful feature in itself and leads to

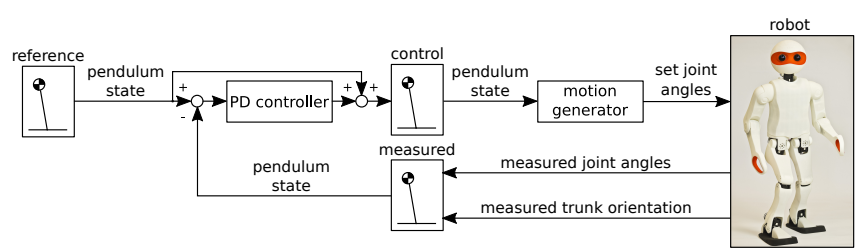

Fig. 8. Control schematic depicting the PD stabilisation. Measurements from the robot are used to calculate an offset pendulum state through PD control on three variables: $\phi_{p}^{B}, \theta_{p}^{B}$ and $l^{B}$. The resulting pendulum state is used as the input for the motion generator.

performing balanced task-oriented motions. An example of this is a kicking motion, where shifting the whole weight of the body onto a single leg, relieves the other one to perform the kick. The trajectory of the foot can then be computed as to hit the ball for a specific result (e.g. side kicks, high kicks, pass kicks and so on). For this motion, only the support coefficient $c_{s}$ value, and desired foot position $F^{R}$ were changed through time. The achieved kick (seen on Fig. 7) is relatively dynamic. The fastest transitions between frames are done in $0.15 \mathrm{~s}$.

Due to the low torque-to-weight ratio in the igus ${ }^{\circledR} \mathrm{Hu}$ manoid Open Platform, the actuators on the robot generally experience difficulties following their reference position, which leads to large CoM tracking errors. This is particularly visible in the ankle joint of the supporting foot. Although the robot can tolerate this to some extent, the precision is decreased. As the motions are generated on-the-fly, we have the possibility to modify them with several corrective actions in response to the CoM tracking error. One of the possibilities is to generate motions with an offset in the desired pendulum projected roll $\phi_{p}^{B}$ and pitch $\theta_{p}^{B}$, as well as compensating for the pendulum extension $l^{B}$. For simplicity, we generate the corrective poses through separate PD regulators on the mentioned variables. A schematic showing the utilised approach can be seen on Fig. 8.

The achieved result in the form of measured CoM with and without PD stabilisation during the kicking motion is shown in Fig. 9. When performing the motion open-loop, the steadystate error was in the range of $3 \mathrm{~cm}$. After lifting the foot, the robot had a tendency to lean towards the kicking foot, and the disturbance of making contact between the foot and the ball led to CoM tracking errors of almost $12 \mathrm{~cm}$. These errors can be attributed to the actuation limitations of the igus ${ }^{\circledR}$ Humanoid Open Platform, where the ankle actuators are underpowered. After applying PD stabilisation on the reference upright-oriented pendulum, the movement of the CoM was much more contained and symmetric. On average, the error was in the range of $2 \mathrm{~cm}$ and did not exceed $5 \mathrm{~cm}$. The steady-state error also decreased. Applying the feedback mechanism also allowed the robot to reject a moderate level of disturbances, which was not possible otherwise.

\section{CONCLUSIONS}

In this work, we presented an analytic, geometric motion generation method for humanoid robots, based on body mass distribution. The statically balanced motions are produced from a small set of directly comprehensible parameters in a 

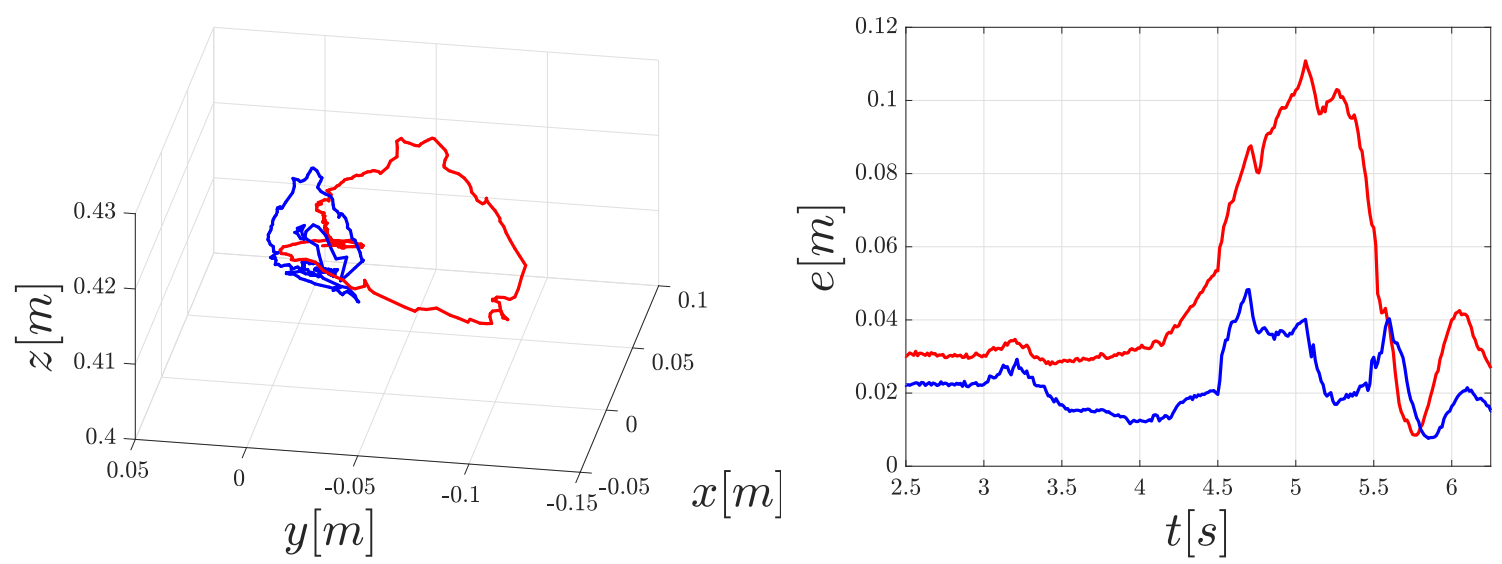

Fig. 9. measured CoM movement (left) and tracking error (right) while kicking. Red represents the open-loop motion, while blue shows the same motion with PD stabilisation enabled.

frame-by-frame manner at interactive rates. As the approach does not rely on any force or torque information, it is applicable to platforms which lack the necessary sensing and actuation capabilities for state-of-the-art Whole-Body Control algorithms. We have experimentally verified the method on the igus ${ }^{\circledR}$ Humanoid Open Platform and were able to produce stable kicking motions which was previously not possible without meticulous design and extensive tuning. One of the most notable merits of the approach is its extendability, which we demonstrated by adding simple PD stabilisation mechanisms on the body pendulum. In future work, feedback mechanisms can be further extended and involve for example, changing the trunk and limb placement strategies. As a pendulum is an integral part of the approach, a whole class of model-based control techniques can be applied to control the robot. The motion generator then is used to tie the controlled pendulum state with a wholebody pose, ensuring that the CoM is accurately tracked. This makes the proposed motion generator a powerful building block for more sophisticated control schemes.

\section{REFERENCES}

[1] K. Hirai, M. Hirose, Y. Haikawa, and T. Takenaka, "The Development of Honda Humanoid Robot," in Robotics and Automation (ICRA), IEEE International Conference on, vol. 2, 1998, pp. 1321-1326.

[2] Y. Fujimoto, A. Kawamura et al., "Simulation of an Autonomous Biped Walking Robot Including Environmental Force Interaction." Institute of Electrical and Electronics Engineers, 2002.

[3] S. Kajita, F. Kanehiro, K. Kaneko, K. Fujiwara, K. Harada, K. Yokoi, and H. Hirukawa, "Resolved Momentum Control: Humanoid Motion Planning based on the Linear and Angular Momentum," in Intelligent Robots and Systems (IROS), IEEE/RSJ International Conference on, vol. 2, 2003, pp. 1644-1650.

[4] L. Sentis and O. Khatib, "Synthesis of Whole-Body Behaviors through Hierarchical Control of Behavioral Primitives," International Journal of Humanoid Robotics, vol. 2, no. 04, pp. 505-518, 2005.

[5] A. Del Prete, F. Romano, L. Natale, G. Metta, G. Sandini, and F. Nori, "Prioritized Optimal Control," in Robotics and Automation (ICRA), IEEE International Conference on, 2014.
[6] A. Herzog, L. Righetti, F. Grimminger, P. Pastor, and S. Schaal, "Balancing experiments on a torque-controlled humanoid with hierarchical inverse dynamics," in Intelligent Robots and Systems (IROS), IEEE/RSJ International Conference on, 2014.

[7] S. Feng, E. Whitman, X. Xinjilefu, and C. G. Atkeson, "Optimizationbased Full Body Control for the DARPA Robotics Challenge," Journal of Field Robotics, vol. 32, no. 2, pp. 293-312, 2015.

[8] H. Dai, A. Valenzuela, and R. Tedrake, "Whole-body Motion Planning with Simple Dynamics and Full Kinematics," in Humanoid Robots (Humanoids), 14th IEEE-RAS International Conference on, 2014.

[9] S. Kajita, F. Kanehiro, K. Kaneko, K. Yokoi, and H. Hirukawa, "The 3d linear inverted pendulum mode: A simple modeling for a biped walking pattern generation," in Intelligent Robots and Systems, 2001. Proceedings. 2001 IEEE/RSJ International Conference on, vol. 1, 2001, pp. 239-246.

[10] S.-H. Lee and A. Goswami, "Reaction Mass Pendulum (RMP): An explicit model for centroidal angular momentum of humanoid robots," in Robotics and Automation (ICRA), IEEE International Conference on, 2007, pp. 4667-4672.

[11] T. Takenaka, T. Matsumoto, and T. Yoshiike, "Real time motion generation and control for biped robot-1 st report: Walking gait pattern generation," in Intelligent Robots and Systems (IROS), IEEE/RSJ International Conference on, 2009, pp. 1084-1091.

[12] M. Vukobratović and B. Borovac, "Zero-Moment Point - Thirty Five Years of its Life," International Journal of Humanoid Robotics, vol. 1, no. 01 , pp. 157-173, 2004.

[13] K. Harada, S. Kajita, K. Kaneko, and H. Hirukawa, "ZMP Analysis for Arm/Leg Coordination," in IROS, 2003, pp. 75-81.

[14] H. Hirukawa, S. Hattori, K. Harada, S. Kajita, K. Kaneko, F. Kanehiro, K. Fujiwara, and M. Morisawa, "A Universal Stability Criterion of the Foot Contact of Legged Robots-Adios ZMP," in Robotics and Automation (ICRA), IEEE International Conference on, 2006, pp. 1976-1983.

[15] R. Drillis, R. Contini, and M. Bluestein, Body segment parameters. New York University, School of Engineering and Science Research Division, NY, 1966.

[16] P. Allgeuer and S. Behnke, "Fused Angles: A representation of body orientation for balance," in Int. Conf. on Intelligent Robots and Systems (IROS), 2015

[17] P. Allgeuer. (2014) Matlab/Octave Rotations Library. [Online]. Available: https://github.com/AIS-Bonn/matlab_octave_rotations_lib/

[18] P. Allgeuer, H. Farazi, M. Schreiber, and S. Behnke, "Child-sized $3 \mathrm{~d}$ printed igus humanoid open platform," in Humanoid Robots $\mathrm{Hu}$ manoids), 2015 IEEE-RAS 15th International Conference on, 2015, pp. 33-40. 